\title{
Ozonioterapia como coadjuvante no tratamento da periodontite: revisão de
}

\section{literatura}

\author{
Ozonetherapy as a supporting in the treatment of periodontitis: literature review \\ Ozoneterapia como coadyuvante en el tratamiento de la periodontitis: revisión de la literatura
}

Recebido: 08/11/2021 | Revisado: 15/11/2021 | Aceito: 16/11/2021 | Publicado: 26/11/2021

Tainara Dutra Silva

ORCID: https://orcid.org/0000-0002-8212-5987

Faculdade Independente do Nordeste, Brasil

E-mail: tainara.bidu1@gmail.com

Daniel Felipe Fernandes Paiva

ORCID: https://orcid.org/0000-0003-4186-9856 Universidade Estadual de Campinas, Brasil E-mail: d265738@ dac.unicamp.br

Dilma Carvalho Santana

ORCID: https://orcid.org/0000-0002-5771-1562

Faculdade Independente do Nordeste, Brasil

E-mail: dilma@fainor.com.br

\begin{abstract}
Resumo
Introdução: A periodontite é caracterizada por ser uma inflamação crônica e multifatorial que está relacionada ao desequilíbrio do biofilme. O padrão-ouro na terapêutica se dá por meio da raspagem periodontal, auxiliada, em geral, à associação de antibióticos tópicos e sistêmicos. Entretanto, os fármacos podem levar ao desenvolvimento de resistência bacteriana ou desconforto do paciente. Desse modo, a ozonioterapia vem se destacando como forma de intervenção adjuvante da doença e do seu processo inflamatório. Objetivo: Evidenciar, com base na literatura, a eficiência da ozonioterapia como coadjuvante no tratamento da periodontite. Metodologia: Uma busca nas bases de dados Pubmed, Embase, Scopus, Web of Science (todas as bases de dados) e Cochrane Library foi realizada utilizando uma estratégia de busca baseada nos termos MeSH "periodontitis", "ozone" e "inflamation" e seus entry terms correspondentes. Nenhum filtro de pesquisa adicional foi aplicado. Foram incluídos apenas artigos com seu texto completo publicado e que tratassem da periodontite, como foco primário do estudo. Os dados foram analisados por meio de síntese qualitativa dos resultados. Resultados e Discussão: Foram selecionados 21 artigos e os mesmos apresentaram desenhos variados. Todos os artigos são enfáticos quanto ao uso seguro da ozonioterapia, entretanto, diferem quanto a seu real efeito prático na clínica. Os estudos ainda não são conclusivos a respeito do efeito esperado. Conclusão: Apesar dos efeitos antiinflamatórios, a ozonioterapia parece não ter efeito significativo como tratamento periodontal, mas pode ser utilizada como medida auxiliar em casos de difícil controle os quais somente a raspagem periodontal, não apresenta resultados satisfatórios.
\end{abstract}

Palavras-chave: Periodontite; Ozonioterapia; Ozônio; Inflamação; Terapêutica.

\begin{abstract}
Introduction: Periodontitis is characterized as a chronic and multifactorial inflammation that is related to biofilm imbalance. The gold standard in therapy is through periodontal scaling, generally supported by an association of common and systemic antibiotics. However, drugs can lead to the development of bacterial resistance or patient discomfort. Thus, ozone therapy has stood out as a form of adjuvant intervention for the disease and the inflammatory process. Objective: To demonstrate, based on the literature, the efficiency of ozone therapy as an adjunct in the treatment of periodontitis. Methodology: A search in Pubmed, Embase, Scopus, Web of Science (all databases), and Cochrane Library databases was performed using a search strategy based on the MeSH terms "periodontitis", "ozone" and "inflammation" and their corresponding input terms. No additional search filters were added. Only articles with their full text published and dealing with periodontitis as the primary focus of the study were included. Data were obtained through a qualitative synthesis of the results. Results and Discussion: 21 articles were selected and similar designs varied. All articles are emphatic about the safe use of ozone therapy, however, they differ as to its real practical effect in the clinic. Studies are still not conclusive about the expected effect. Conclusion: Despite its antiinflammatory effects, ozone therapy does not seem to have a significant effect as periodontal treatment, but it can be used as an auxiliary measure in difficult-to-control cases in which periodontal scaling alone does not present satisfactory results.
\end{abstract}

Keywords: Periodontitis, Ozone therapy, Ozone, Inflammation, Therapeutics. 


\begin{abstract}
Resumen
Introducción: La periodontitis se caracteriza por ser una inflamación crónica y multifactorial relacionada con el desequilibrio del biofilm. El estándar de oro en la terapia es a través del raspado periodontal, ayudado, en general, por la asociación de antibióticos tópicos y sistémicos. Sin embargo, los medicamentos pueden provocar el desarrollo de resistencia bacteriana o malestar del paciente. Así, la ozonoterapia se ha destacado como una forma de intervención adyuvante de la enfermedad y su proceso inflamatorio. Objetivo: Demostrar, con base en la literatura, la eficacia de la ozonoterapia como coadyuvante en el tratamiento de la periodontitis. Metodología: Se realizó una búsqueda en Pubmed, Embase, Scopus, Web of Science (todas las bases de datos) y en las bases de datos de la Biblioteca Cochrane utilizando una estrategia de búsqueda basada en los términos MeSH "periodontitis", "ozono" e "inflamación” y sus correspondientes términos de entrada. No se aplicó ningún filtro de búsqueda adicional.Solo se incluyeron artículos con su texto completo publicado y que trataran de periodontitis como el foco principal del estudio. Los datos se analizaron mediante una síntesis cualitativa de los resultados. Resultados y Discusión: Se seleccionaron 21 artículos que presentaron diseños variados. Todos los artículos son enfáticos sobre el uso seguro de la ozonoterapia, sin embargo, difieren en cuanto a su efecto práctico real en la clínica. Los estudios aún no son concluyentes sobre el efecto esperado. Conclusión: a pesar de sus efectos antiinflamatorios, la ozonoterapia no parece tener un efecto significativo como tratamiento periodontal, pero puede utilizarse como medida auxiliar en casos de difícil control en los que el raspado periodontal por sí solo no presenta resultados satisfactorios.
\end{abstract}

Palabras clave: Periodontitis; terapia de ozono; Ozono; Inflamación; Terapéutica.

\title{
1. Introdução
}

A periodontite caracteriza-se por ser uma doença inflamatória crônica e multifatorial, a qual está relacionada ao desequilíbrio do biofilme disbiótico, caracterizando-se pela destruição progressiva do aparelho de suporte dentário. Sua definição abarca o sangramento gengival, presença de bolsa periodontal, perda de suporte dos tecidos do periodonto e deformação do osso alveolar. Essa enfermidade constitui um importante problema de saúde pública devido à sua alta prevalência, perda e incapacidade dentária, o que gera um comprometimento da função mastigatória e estética, sendo fonte de preconceitos e, consequentemente, desigualdade social. Além disso, a periodontite gera custos significativos com tratamentos dentários e tem um impacto expressivo na saúde geral do indivíduo. Ademais, sua prevalência tem sido investigada em combinação com condições sistêmicas como, gravidez, doenças cardiovasculares, diabetes mellitus, doenças respiratórias, doença renal crônica e síndrome metabólica (Fischer et al., 2020; Papanou et al., 2018).

Acerca do tratamento da periodontite, seu sucesso a curto e longo prazo depende da ruptura mecânica do biofilme aderido à estrutura dental. De fato, a instrumentação subgengival (ou raspagem) é considerada o padrão ouro da terapia periodontal, sendo, dividida nas etapas de desbridamento (remoção ou ruptura da estrutura biológica contaminada) e alisamento radicular, que consiste na remoção do cemento ou dentina superficial com cálculo, toxinas ou microrganismos contaminantes (Graziani et al., 2017).

Além do tratamento mecânico, podem ser administrados, antissépticos, como: clorexidina, tetraciclina, doxiciclina de liberação lenta, minociclina para irrigação subgengival e controle do biofilme. No entanto, alguns desses produtos são tecnicamente difíceis de manusear, pois são rapidamente retirados das bolsas periodontais, além do alto custo e possibilidade de desenvolverem resistência bacteriana. No caso da clorexidina, seus efeitos adversos incluem, coloração extrínseca marrom nos dentes, língua e restaurações, alterações na percepção do paladar e aumento da deposição de cálculo (Domb, 2014; Graziani et al., 2017; Herrera,et., al 2008).

Devido aos problemas decorrentes ao uso de antibióticos como adjuvantes no tratamento da periodontite, a ozonioterapia vem se destacando como uma opção de intervenção no controle da doença, uma vez que demonstrou ser eficaz na eliminação de determinados grupos bacterianos, bem como de vírus e fungos aderidos, à estrutura dental (Gupta \& Mansi, 2012).

A ozonioterapia passou a ser utilizada como uma alternativa ao tratamento da periodontite, em razão do seu potencial antimicrobiano e possibilidade de controle do processo inflamatório local (Uraz et al., 2019; Khan et al., 2019).

A opção terapêutica deriva-se das características do ozônio como um importante agente oxidante. No contexto da 
periodontite, a maioria da microbiota patogênica é anaeróbica. Portanto, o uso de um agente oxidativo permitiria a redução de atividade bacteriana e possibilitaria, desse modo, uma modulação da patogenia da doença. (Deepthi \& Bilichodmath, 2020; Gupta \& Mansi, 2012; Tasdemir et al., 2019).

A administração do ozônio pode se dar, por meio do gás propriamente dito, além de água e óleos ozonizados. Tais apresentações, caracterizam-se pela boa difusão tecidual e atingem regiões de maiores profundidades, como as bolsas periodontais. Esta característica permite ao ozônio atuar em regiões de resistência bacteriana clássica, como as zonas do ápice dentário. Desse modo, a ozonioterapia pode eliminar as bactérias que colonizam essa região e reduzir os produtos de resíduos tóxicos que impedem efetivamente a cura completa das estruturas locais, além de acelerar significativamente o processo de cicatrização (Gupta \& Mansi, 2012). Cerca de 25\% das lesões tratadas com essa intervenção não parecem ter recorrência, sinalizando que o poder desinfetante indiscriminado desse gás, parece ofuscar o uso de antibióticos (Domb, 2014).

Com base nessas informações, a presente revisão de literatura buscou evidenciar o uso da ozonoterapia, como tratamento adjuvante para o controle e da periodontite.

\section{Metodologia}

Trata-se de uma revisão do tipo narrativa da literatura, a qual busca sintetizar o conhecimento a respeito de uma temática, possibilitando, desse modo, a existência de uma odontologia baseada em evidência. O protocolo utilizado teve como base o trabalho de Honório \& Santiago Júnior (2021).

O estudo foi conduzido por meio da seguinte pergunta: “A ozonioterapia é eficaz como coadjuvante no tratamento da periodontite?" Para a análise dos dados foram considerados a eficácia do ozônio, promoção da boa reparação tecidual, pacientes com periodontite (como população afetada) e modulação da inflamação, como a resposta clínica esperada. Como desfecho primário, foram avaliados métodos de avaliação da inflamação, tais como: índice de sangramento gengival, índice de sangramento a sondagem, profundidade de sondagem e níveis de interleucinas inflamatórias.

A busca foi realizada nas seguintes bases de dados: Pubmed, Embase, Scopus, Web of Science e Cochrane Library. Foi utilizada a seguinte estratégia de busca: "(("Periodontitis" OR "Periodontitides" OR "Pericementitis" OR "Pericementitides") AND (“Ozone” OR “Ozonetherapy" OR "Ozone-therapy” OR "Low Level Ozone” OR "Level Ozone, Low" OR "Ozone, Low Level” OR "Ground Level Ozone" OR "Level Ozone, Ground" OR "Ozone, Ground Level”) AND ("Inflammation" OR "Inflammations" OR "Innate Inflammatory Response" OR "Inflammatory Response, Innate" OR "Innate Inflammatory Responses"))". Ressalta-se que na base de dados EMBASE, os descritores MeSH foram substituídos pelos seus correspondentes Emtree Terms, como sugere a própria ferramenta de busca.

As buscas resgataram artigos independentes de sua data de publicação ou desenho de estudo. Foram avaliados somente trabalhos que estivessem escritos nos idiomas português, inglês e espanhol, de modo a possibilitar um correto aprofundamento crítico pelos revisores. Ademais, para critérios de inclusão, houve a necessidade do foco clínico apresentado pelo artigo, corresponder com algum nível de inflamação periodontal. Ainda que, a ozonioterapia tenha sido utilizada, pelo menos, como uma das alternativas de tratamento. Somente as pesquisas publicadas com seu texto completo foram analisadas, de modo a possibilitar o aprofundamento temático exigido.

Para a sumarização do conteúdo, optou-se pela síntese dos relatos a respeito do tema. Desse modo, uma busca primária foi utilizada e foram excluídos os estudos resgatados sem relação com a temática proposta pelos seus títulos, resumos ou palavras-chave. Após a seleção dos trabalhos considerados relevantes, efetuou-se a leitura completa dos relatos e a avaliação de seu conteúdo.

Após a leitura, os artigos foram sumarizados em software Excel ${ }^{\circledR}$ (Microsoft Corporation $®$ ) contendo as seguintes informações: autor e ano, objetivo e desfecho. Tais considerações auxiliaram a correlação dos textos e guiaram a síntese 
qualitativa dos resultados como utilizado na síntese de Oliveira et al (2020). Após essa etapa, os dados coletados foram cruzados com demais referências sobre periodontite e ozonioterapia, de modo a realizar uma reflexão ainda mais abrangente da temática.

\section{Resultados}

Foram resgatados 21 artigos segundo os critérios previamente estabelecidos. Desses, 16 demonstraram resultados positivos para a relação entre o uso de ozônio e o controle eficaz da doença periodontal. Os demais, constataram que o efeito da ozonioterapia não influencia positivamente no avanço da periodontite. É válido destacar que nenhum estudo relatou que o a terapia com ozônio, comprometeu a saúde do paciente. Os achados foram sumarizados e podem ser verificados no Quadro 1, contendo título do manuscrito, autor e ano de publicação, objetivos e principais resultados.

Quadro 1. Sumarização dos achados dos artigos selecionados.

\begin{tabular}{|c|c|c|c|}
\hline Título & Autor e Ano & Objetivos & Resultados/Conclusão \\
\hline \multirow{2}{*}{$\begin{array}{l}\text { Ozone therapy in endodontic treatment } \\
\text { of chronic apical periodontitis }\end{array}$} & \multirow{2}{*}{$\begin{array}{l}\text { Gusiyska e } \\
\text { Kisselova, } \\
2010 .\end{array}$} & $\begin{array}{l}\text { 1. Analisar a eficácia do } \mathrm{NaOCl} \\
\text { ozonizado para irrigação em } \\
\text { tratamento endodôntico. }\end{array}$ & $\begin{array}{l}\mathrm{O} \mathrm{NaOCl} \text { ozonizado mostrou resultados } \\
\text { positivos quanto a sua eficácia e potência. }\end{array}$ \\
\hline & & $\begin{array}{l}\text { 2. Avaliar o processo de cicatrização } \\
\text { utilizando a solução irrigadora em fase } \\
\text { final do tratamento endodôntico. }\end{array}$ & $\begin{array}{l}\text { A ozonioterapia e o líquido ozonizado } \\
\text { aumentam a potência de descontaminação } \\
\text { final do espaço endodôntico e periapical. }\end{array}$ \\
\hline \multirow{3}{*}{$\begin{array}{l}\text { Evaluating clinical and laboratory } \\
\text { effects of ozone in non-surgical } \\
\text { periodontal treatment: a randomized } \\
\text { controlled trial }\end{array}$} & \multirow{3}{*}{$\begin{array}{l}\text { Dengizek., et al, } \\
2019\end{array}$} & \multirow{3}{*}{$\begin{array}{l}\text { 1. Determinar os efeitos clínicos e } \\
\text { bioquímicos do uso do ozônio gasoso } \\
\text { acompanhado da raspagem radicular } \\
\text { em pacientes com periodontite crônica. }\end{array}$} & $\begin{array}{l}\text { As mudanças se mostraram semelhantes com } \\
\text { o uso do ozônio gasoso. }\end{array}$ \\
\hline & & & $\begin{array}{c}\text { O tratamento com SRP mais ozônio foi } \\
\text { considerado irrelevante quanto ao SRP } \\
\text { sozinho. }\end{array}$ \\
\hline & & & $\begin{array}{l}\text { Não houve melhora significativa na } \\
\text { recuperação periodontal. }\end{array}$ \\
\hline \multirow{4}{*}{$\begin{array}{c}\text { Effect of Aqueous Ozone on the NF-kB } \\
\text { System }\end{array}$} & \multirow{4}{*}{$\begin{array}{l}\text { Huth., et al, } \\
2007 .\end{array}$} & \multirow{2}{*}{$\begin{array}{l}\text { 1. Investigar o efeito do ozônio aquoso } \\
\text { considerando sua influência } \\
\text { antibacteriana. }\end{array}$} & $\begin{array}{l}\text { Após utilização do ozônio aquoso nas } \\
\text { superfícies orais estimuladas com TNF obteve } \\
\text { suas atividades inibidas. }\end{array}$ \\
\hline & & & $\begin{array}{l}\text { As atividades do NF- } \mathrm{kB} \text { foram impedidas } \\
\text { após a incubação dessas células com meio } \\
\text { ozonizado. }\end{array}$ \\
\hline & & \multirow{2}{*}{$\begin{array}{l}\text { 2. Associar a água ozonizada no } \\
\text { sistema NF- kB para sinalização } \\
\text { relacionada à inflamação. }\end{array}$} & $\begin{array}{l}\text { Comprovou-se que os aminoácidos } \\
\text { ozonizados característicos são principais para } \\
\text { a inibição. }\end{array}$ \\
\hline & & & $\begin{array}{l}\text { O ozônio desempenha capacidade anti- } \\
\text { inflamatória. }\end{array}$ \\
\hline \multirow{3}{*}{$\begin{array}{l}\text { The Effect of Gaseous Ozone Therapy } \\
\text { in Conjunction with Periodontal } \\
\text { Treatment on Glycated Hemoglobin } \\
\text { Level in Subjects with Type } 2 \text { Diabetes } \\
\text { Mellitus: An Unmasked Randomized } \\
\text { Controlled Trial }\end{array}$} & \multirow{3}{*}{$\begin{array}{l}\text { Rapone., et al, } \\
2020 .\end{array}$} & $\begin{array}{l}\text { 1. Comparar a eficácia clínica de uma } \\
\text { intervenção periodontal intensiva. }\end{array}$ & $\begin{array}{l}\text { O tratamento periodontal em conjunto ao } \\
\text { tratamento com gás ozônio não apresentou } \\
\text { mudança significativa. }\end{array}$ \\
\hline & & \multirow{2}{*}{$\begin{array}{c}\text { 2. Associar o tratamento periodontal } \\
\text { convencional em conjunto a terapia } \\
\text { ozonizada na redução do nível de } \\
\text { hemoglobina glicada. }\end{array}$} & $\begin{array}{l}\text { Apesar das mudanças não terem sidos } \\
\text { significativas no tratamento periodontal, o } \\
\text { uso do ozônio tendeu a reduzir os níveis de } \\
\text { hemoglobina glicada. }\end{array}$ \\
\hline & & & $\begin{array}{l}\text { A terapia ozonizada mostra uma vantagem } \\
\text { comparada ao tratamento periodontal } \\
\text { convencional. }\end{array}$ \\
\hline \multirow{2}{*}{$\begin{array}{l}\text { Comparative evaluation of the effect of } \\
\text { Ozone therapy and Photodynamic } \\
\text { therapy in non-surgical management of } \\
\text { Chronic periodontitis: A split mouth } \\
\text { longitudinal study }\end{array}$} & \multirow{2}{*}{$\begin{array}{l}\text { Ameyaroy., et } \\
\quad \text { al, } 2021\end{array}$} & $\begin{array}{l}\text { 1. Avaliar o efeito do ozônio aquoso } \\
\text { em conjunção a terapia fotodinâmica. }\end{array}$ & $\begin{array}{l}\text { A terapia com ozônio alcançou resultados } \\
\text { relevantes quanto a redução da profundidade } \\
\text { das bolsas periodontais. }\end{array}$ \\
\hline & & $\begin{array}{l}\text { 2. Determinar a eficácia da } \\
\text { ozonioterapia no tratamento não } \\
\text { cirúrgico da periodontite crônica. }\end{array}$ & $\begin{array}{c}\text { A decorrência dos tratamentos obteve } \\
\text { resultados semelhantes nos primeiros } 2 \\
\text { meses. }\end{array}$ \\
\hline
\end{tabular}




\begin{tabular}{|c|c|c|c|}
\hline & & & $\begin{array}{l}\text { Seguidamente comprovou-se que o } \\
\text { tratamento com OT teve um aumento gradual } \\
\text { em comparação ao tratamento com PDT. }\end{array}$ \\
\hline \multirow{3}{*}{ Ozone Application in Dentistry } & \multirow{3}{*}{$\begin{array}{l}\text { William C } \\
\text { Domb, } 2014\end{array}$} & \multirow{3}{*}{$\begin{array}{l}\text { 1. Julgar a eficácia da aplicação do } \\
\text { ozônio nos campos da odontologia. }\end{array}$} & $\begin{array}{c}\text { Acerca dessa terapia os resultados não são } \\
\text { harmônicos em todos os estudos } \\
\text { apresentados. }\end{array}$ \\
\hline & & & $\begin{array}{l}\text { A utilização do ozônio gasoso tem impacto } \\
\text { maior quanto a diminuição das bactérias } \\
\text { facilitando a cicatrização. }\end{array}$ \\
\hline & & & $\begin{array}{l}\text { Por fim foi determinado que o uso do ozônio } \\
\text { é indicado na descontaminação e na } \\
\text { descolonização das bactérias em meio bucal. }\end{array}$ \\
\hline \multirow{2}{*}{$\begin{array}{l}\text { Evaluation of the influence of } \\
\text { ozonotherapy on the clinical parameters } \\
\text { and MMP levels in patients with } \\
\text { chronic and aggressive periodontitis }\end{array}$} & \multirow{2}{*}{$\begin{array}{l}\text { Skurska., et al, } \\
2010 .\end{array}$} & \multirow{2}{*}{$\begin{array}{l}\text { 1. Conferir a condição clínica com o } \\
\text { nível salivar isolado e ozonioterapia } \\
\text { em pacientes com periodontite crônic e } \\
\text { agressiva. }\end{array}$} & $\begin{array}{l}\text { Todos os critérios clínicos apresentados } \\
\text { tiveram uma diminuição expressiva depois do } \\
\text { tramento. }\end{array}$ \\
\hline & & & $\begin{array}{c}\text { Acerca do tratamento com ozônio não teve } \\
\text { uma maior melhora quanto aos parâmetros } \\
\text { clínicos periodontais, nem nos níveis de } \\
\text { metaloproteinases }\end{array}$ \\
\hline \multirow{3}{*}{$\begin{array}{l}\text { Application of ozone in the treatment of } \\
\text { periodontal disease }\end{array}$} & \multirow{3}{*}{$\begin{array}{l}\text { Srikanth, } \\
\text { Sathish, Harsha, } \\
\text { 2013. }\end{array}$} & $\begin{array}{l}\text { 1. Oferecer conhecimentos sobre as } \\
\text { aplicações clínicas do ozônio. }\end{array}$ & $\begin{array}{l}\text { Apresenta-se sinais de que existe } \\
\text { biocompatibilidade in vitro do uso de água } \\
\text { ozonizada com células orais humanas. }\end{array}$ \\
\hline & & \multirow[b]{2}{*}{$\begin{array}{l}\text { 2. Sintetizar os estudos in vitro a } \\
\text { serviço da periodontia onde são } \\
\text { utilizadas aplicações de ozônio. }\end{array}$} & $\begin{array}{l}\text { O uso do ozônio é uma prática fácil de } \\
\text { manusear e indolor. }\end{array}$ \\
\hline & & & $\begin{array}{l}\text { A compatibilidade da ozonioterapia em } \\
\text { células de fibroblastos gengivais e células } \\
\text { periodontais faz com que o seu uso seja de } \\
\text { bastante potencial na odontologia. }\end{array}$ \\
\hline \multirow{3}{*}{$\begin{array}{l}\text { The Study of Ozone Ointment on } \\
\text { Human Gingival Fibroblasts Cell } \\
\text { Proliferation Ability and Anti- } \\
\text { Inflammatory }\end{array}$} & \multirow{3}{*}{$\begin{array}{l}\text { Wang., et al, } \\
2018\end{array}$} & $\begin{array}{c}\text { 1. Verificar os efeitos da exposição ao } \\
\text { ozônio em pomada na produção de } \\
\text { colágeno. }\end{array}$ & $\begin{array}{l}\text { O uso do ozônio não apresentou nenhuma } \\
\text { ação citotóxica. }\end{array}$ \\
\hline & & \multirow{2}{*}{$\begin{array}{l}\text { 2. Averiguar o resultado do tratamento } \\
\text { com ozônio em citocinas pró- } \\
\text { inflamatórias e em fibroblastos } \\
\text { gengivais in vitro. }\end{array}$} & $\begin{array}{l}\text { A utilização da pomada ozonizada } \\
\text { proporcionou um aumento significativo na } \\
\text { produção de colágeno nas citocinas HGFs. }\end{array}$ \\
\hline & & & $\begin{array}{l}\text { Consequentemente, o uso da pomada fez com } \\
\text { que diminuísse a liberação das citocinas, } \\
\text { sugerindo que o efeito terapêutico do ozônio } \\
\text { contra a doença periodontal é em parte devido } \\
\text { à modulação da função do HGFs. }\end{array}$ \\
\hline \multirow{2}{*}{$\begin{array}{l}\text { Clinical and microbiological effects of } \\
\text { ozone nano-bubble water irrigation as } \\
\text { an adjunct to mechanical subgingival } \\
\text { debridement in periodontitis patients in } \\
\text { a randomized controlled trial }\end{array}$} & \multirow{2}{*}{$\begin{array}{l}\text { Hayakumo., et } \\
\quad \text { al, } 2012\end{array}$} & $\begin{array}{l}\text { 1. Avaliar o efeito de nanobolhas } \\
\text { (NBW3) de ozônio para tratamento } \\
\text { periodontal. }\end{array}$ & $\begin{array}{l}\text { Determinou-se que o uso de irrigação com } \\
\text { NBW3 é um acréscimo bastante proveitoso } \\
\text { para o tratamento periodontal. }\end{array}$ \\
\hline & & $\begin{array}{l}\text { 2. Examinar o nível de segurança e } \\
\text { estabilidade dos seus impactos } \\
\text { antimicrobianos. }\end{array}$ & $\begin{array}{c}\text { Por possuir alta taxa antimicrobiana, o } \\
\text { tratamento com nanobolhas foi considerado } \\
\text { indispensável como adjuvante no controle da } \\
\text { doença periodontal. }\end{array}$ \\
\hline \multirow{2}{*}{$\begin{array}{l}\text { Clinical utility of ozone therapy in } \\
\text { dental and oral medicine }\end{array}$} & \multirow{2}{*}{ Suh., et al, 2019} & \multirow{2}{*}{$\begin{array}{l}\text { 1. Revisar a parte química e as } \\
\text { diferentes formas de aplicação do } \\
\text { ozônio em odontologia. }\end{array}$} & $\begin{array}{l}\text { A terapia com ozônio foi considerada } \\
\text { necessária na eliminação de microrganismos } \\
\text { aderidos à superfície dentária. }\end{array}$ \\
\hline & & & $\begin{array}{l}\text { O estudo com ozônio comprovou que o uso } \\
\text { de sua terapia tem caráter positivo no } \\
\text { tratamento de patologias orais. }\end{array}$ \\
\hline \multirow{2}{*}{$\begin{array}{l}\text { Efficacy of gaseous ozone in smoking } \\
\text { and non-smoking gingivitis patients }\end{array}$} & \multirow{2}{*}{$\begin{array}{c}\text { Talmaç, Çaliş̧ir., } \\
2020 .\end{array}$} & $\begin{array}{l}\text { 1. Investigar os efeitos da } \\
\text { ozonioterapia e sua aplicação nos } \\
\text { tecidos gengivais. }\end{array}$ & $\begin{array}{l}\text { Apresentou-se uma melhora significativa na } \\
\text { diminuição da patologia. }\end{array}$ \\
\hline & & $\begin{array}{l}\text { 2. Apurar informações a respeito da } \\
\text { terapêutica utilizando ozônio gasoso } \\
\text { nas bolsas periodontais em pacientes } \\
\text { fumantes e não fumantes. }\end{array}$ & $\begin{array}{l}\text { O ozônio é considerado relevante para } \\
\text { tratamentos gengivais e periodontais por } \\
\text { fornecer valiosas contribuições para melhora } \\
\text { no grau da doença. }\end{array}$ \\
\hline
\end{tabular}




\begin{tabular}{|c|c|c|c|}
\hline \multirow{2}{*}{$\begin{array}{l}\text { Ozonated oil in wound healing: What } \\
\text { has already been proven? }\end{array}$} & \multirow{2}{*}{$\begin{array}{l}\text { Anzolin, } \\
\text { Kaross, Bertol., } \\
2020 .\end{array}$} & $\begin{array}{l}\text { 1. Apresentar o óleo ozonizado como } \\
\text { possível tratamento de feridas. }\end{array}$ & $\begin{array}{l}\text { Foi constatado que o uso do ozônio em óleo } \\
\text { promove uma boa cicatrização de inflamações } \\
\text { agudas e crônicas. }\end{array}$ \\
\hline & & $\begin{array}{l}\text { 2. Avaliar as propriedades bactericidas } \\
\text { e antifúngicas como recursos } \\
\text { terapêuticos em inflamações orais. }\end{array}$ & $\begin{array}{c}\text { Possui efeitos benéficos em relação a redução } \\
\text { das bactérias em meio oral e rápida reparação } \\
\text { tecidual. }\end{array}$ \\
\hline \multirow{3}{*}{$\begin{array}{c}\text { Statistical evaluation of antimicrobial } \\
\text { influence of medical Ozone as a part of } \\
\text { inflammatory prevention of periodontal } \\
\text { diseases }\end{array}$} & \multirow{3}{*}{$\begin{array}{l}\text { Kubyshkina., et } \\
\text { al, } 2018\end{array}$} & \multirow{3}{*}{$\begin{array}{l}\text { 1. Informar a respeito da intensidade } \\
\text { da água ozonizada no tratamento de } \\
\text { complicações periodontais. }\end{array}$} & $\begin{array}{l}\text { A aplicação do ozônio aquoso fornece uma } \\
\text { maior eficiência terapêutica nas doenças } \\
\text { periodontais inflamatórias. }\end{array}$ \\
\hline & & & $\begin{array}{l}\text { Seu uso tende a ser em curto prazo, além de } \\
\text { fornecer a oportunidade a tratamentos em } \\
\text { pacientes intolerantes à farmacoterapia. }\end{array}$ \\
\hline & & & $\begin{array}{c}\text { Comprovado que a ozonioterapia é } \\
\text { confirmada como um método eficaz de } \\
\text { tratamento agudo da doença periodontal } \\
\text { inflamatória. }\end{array}$ \\
\hline \multirow{2}{*}{$\begin{array}{l}\text { The effects of ozone therapy on } \\
\text { periodontal therapy: A randomized } \\
\text { placebo controlled clinical trial }\end{array}$} & \multirow{2}{*}{$\begin{array}{l}\text { Tasdemir ., et } \\
\quad \text { al, } 2019\end{array}$} & \multirow{2}{*}{$\begin{array}{l}\text { 1. Analisar os parâmetros clínicos e } \\
\text { bioquímicos do efeito da ozonioterapia } \\
\text { em pacientes com periodontite } \\
\text { generalizada e grave após terapia } \\
\text { periodontal não cirúrgica. }\end{array}$} & $\begin{array}{l}\text { A terapia com ozônio não obteve nenhum } \\
\text { efeito complementar nos preceitos } \\
\text { periodontais. }\end{array}$ \\
\hline & & & $\begin{array}{l}\text { Apenas no fluido de fenda gengival } \\
\text { pentraxina- } 3 \text { (PTX-3) foram um pouco mais } \\
\text { baixos nos locais de ozônio. }\end{array}$ \\
\hline \multirow{3}{*}{$\begin{array}{l}\text { Efficacy of Ozonised Water and } 0.2 \% \\
\text { Chlorhexidine Gluconate in the } \\
\text { Management of Chronic Periodontitis } \\
\text { when Used as an Irrigant in } \\
\text { Conjugation with Phase I Therapy }\end{array}$} & \multirow{3}{*}{$\begin{array}{l}\text { Kaur., et al, } \\
2019 .\end{array}$} & $\begin{array}{l}\text { 1. Investigar a eficácia da irrigação } \\
\text { com ozônio aquoso e gluconato de } \\
\text { clorexidina } 0,2 \% \text {. }\end{array}$ & $\begin{array}{l}\text { O estudo apresentou uma melhora } \\
\text { significativa nos parâmetros clínicos. }\end{array}$ \\
\hline & & \multirow{2}{*}{$\begin{array}{l}\text { 2. Avaliar o poder da irrigação dessas } \\
\text { substâncias como tratamento adjuvante } \\
\text { em bolsas periodontais. }\end{array}$} & $\begin{array}{l}\text { A água ozonizada apontou ser mais eficaz que } \\
\text { o uso da clorexidina. }\end{array}$ \\
\hline & & & $\begin{array}{l}\text { A irrigação com ozônio aquoso se mostrou } \\
\text { mais propícia do que as terapêuticas } \\
\text { convencionais. }\end{array}$ \\
\hline \multirow{3}{*}{$\begin{array}{l}\text { Evaluation of ozone as an adjunct to } \\
\text { scaling and root planing in the treatment } \\
\text { of chronic periodontitis: A randomized } \\
\text { clinico-microbial study }\end{array}$} & \multirow{3}{*}{$\begin{array}{l}\text { Vasthavi., et al, } \\
2019\end{array}$} & \multirow{3}{*}{$\begin{array}{c}\text { 1. Analisar o uso da ozonioterapia } \\
\text { como irrigação como adjuvante no } \\
\text { tratamento de periodontite crônica } \\
\text { generalizada. }\end{array}$} & $\begin{array}{c}\text { A análise mostrou uma redução de patógenos } \\
\text { em ambos os grupos com doenças } \\
\text { periodontais. }\end{array}$ \\
\hline & & & $\begin{array}{l}\text { Evidenciou um avanço significativo nos } \\
\text { parâmetros clínicos e microbiológicos. }\end{array}$ \\
\hline & & & $\begin{array}{l}\text { A ozonioterapia é sugerida como um } \\
\text { complemento adjuvante eficiente no } \\
\text { tratamento periodontal. }\end{array}$ \\
\hline \multirow{3}{*}{$\begin{array}{l}\text { Evaluation of Salivary Matrix } \\
\text { Metalloproteinase (MMP-8) in } \\
\text { Periodontal Patients Undergoing Non- } \\
\text { Surgical Periodontal Therapy and } \\
\text { Mouthwash Based on Ozonated Olive } \\
\text { Oil: A Randomized Clinical Trial }\end{array}$} & \multirow{3}{*}{$\begin{array}{l}\text { Nardi., et al, } \\
\quad 2020 .\end{array}$} & \multirow{3}{*}{$\begin{array}{l}\text { 1. Classificar o tratamento periodontal } \\
\text { não cirúrgico com o uso de óleo } \\
\text { ozonizado a base de azeite de oliva } \\
\text { com metaloproteinase salivar (MMP- } \\
\text { 8). }\end{array}$} & $\begin{array}{c}\text { A melhora foi considerada em ambos os } \\
\text { grupos. }\end{array}$ \\
\hline & & & $\begin{array}{l}\text { Deteve um rendimento maior quando } \\
\text { comparado ao azeite de oliva ozonizado na } \\
\text { redução de MMP- } 8 \text {. }\end{array}$ \\
\hline & & & $\begin{array}{l}\text { A união da raspagem com o óleo ozonizado } \\
\text { alcançou mais resultados juntos do que } \\
\text { somente a raspagem. }\end{array}$ \\
\hline \multirow{2}{*}{$\begin{array}{l}\text { Evaluation of the Clinical and } \\
\text { Antimicrobial Effects of the Er:YAG } \\
\text { Laser or Topical Gaseous Ozone as } \\
\text { Adjuncts to Initial Periodontal Therapy }\end{array}$} & \multirow{2}{*}{$\begin{array}{l}\text { Y1lmaz., et al, } \\
2013\end{array}$} & \multirow{2}{*}{$\begin{array}{l}\text { 1. Ponderar decorrências clínicas e } \\
\text { microbiológicas quanto ao tratamento } \\
\text { com laser Er: YAG juntamente com } \\
\text { aplicação tópica de ozônio gasoso em } \\
\text { pacientes com periodontite crônica. }\end{array}$} & $\begin{array}{l}\text { Melhorias clínicas foram observadas dentro } \\
\text { de cada grupo avaliado. }\end{array}$ \\
\hline & & & $\begin{array}{l}\text { Uma diminuição considerável foi constatada } \\
\text { em relação ao uso de ozônio gasoso tópico } \\
\text { exibindo ter um efeito microbiano equivalente } \\
\text { ao do laser Er: YAG. }\end{array}$ \\
\hline
\end{tabular}




\begin{tabular}{|c|c|c|c|}
\hline \multirow{2}{*}{$\begin{array}{l}\text { Evaluation of the effect of topical and } \\
\text { systemic ozone application in } \\
\text { periodontitis: an experimental study in } \\
\text { rats* }\end{array}$} & \multirow{2}{*}{$\begin{array}{l}\text { Saglam., et al, } \\
2019 .\end{array}$} & $\begin{array}{l}\text { 1. Avaliar os efeitos da aplicação } \\
\text { tópica e sistêmica de ozônio gasoso na } \\
\text { perda óssea alveolar. }\end{array}$ & $\begin{array}{l}\text { O uso de ozônio contribui para redução de } \\
\text { células positivas em alterações } \\
\text { histopatológicas e imunohistoquímicas } \\
\text { (RANKL). }\end{array}$ \\
\hline & & $\begin{array}{l}\text { 2. Determinar a eficácia do ozônio nas } \\
\text { células positivas em alterações } \\
\text { histopatológicas. }\end{array}$ & $\begin{array}{l}\text { A aplicação de ozônio sistêmico foi } \\
\text { considerada satisfatória na redução de perda } \\
\text { óssea alveolar. }\end{array}$ \\
\hline \multirow{2}{*}{$\begin{array}{l}\text { Comparison of the amount of bacterial } \\
\text { microcolonies formed on using distilled } \\
\text { water and ozonated water as an } \\
\text { irrigating agent }\end{array}$} & \multirow{2}{*}{$\begin{array}{l}\text { R. Niveda, } \\
\text { Sankari } \\
\text { Malaiappan, } \\
\text { 2019. }\end{array}$} & \multirow{2}{*}{$\begin{array}{l}\text { 1. Investigar o efeito da água } \\
\text { ozonizada como agente irrigante no } \\
\text { tratamento de raspagem radicular. }\end{array}$} & $\begin{array}{l}\text { A aplicação de ozônio gasoso obteve redução } \\
\text { significativa das colônias de bactérias } \\
\text { formadas por condições anaeróbias. }\end{array}$ \\
\hline & & & $\begin{array}{l}\text { A ozonioterapia foi considerada eficaz por } \\
\text { reduzir a quantidade de microorganismos } \\
\text { presentes na doença periodontal. }\end{array}$ \\
\hline
\end{tabular}

Fonte: Autores (2021).

Os trabalhos de caráter positivo variaram seu período de publicação entre 2007 (Huth et al, 2007) e 2020 (Nardi et al, 2020, Talmaç, Çalişir, 2020). Todos os estudos resgatados com desenho do tipo exclusivamente in vitro, modelo animal e revisões apontaram a ozonioterapia como uma medida eficaz de tratamento adjuvante. Ademais, oito estudos de caráter clínico, também estabeleceram uma relação positiva com a terapia complementar. As formas de aplicação variaram, apresentando-se como nanobolhas (Hayakumo et al, 2013), óleo ozonizado (Nardi et al, 2020, Anzolin et al., 2020); água (R. Niveda, Sankari Malaiappan, 2019, Vasthavi., et al, 2019, Kaur., et al, 2019, Kubyshkina., et al, 2018, Hayakumo., et al, 2012, Srikanth et al., 2013, Ameyaroy., et al, 2021, Huth., et al, 2007, Gusiyska e Kisselova, 2010); e na forma gasosa (Saglam., et al, 2019, Yılmaz., et al, 2013, William C Domb, 2014, Rapone., et al, 2020., Dengizek., et al, 2019).

Todos os estudos que não encontraram diferença significativa entre o uso da ozonioterapia e a doença periodontal tinham caráter clínico. Variaram, com relação ao tempo de publicação, entre 2010 (Skurska et al, 2010) e 2020 (Rapone et al, 2020). Todos os artigos utilizaram a aplicação tópica de ozônio gasoso. É válido ressaltar que, apesar de o estudo de Rapone et al, 2020, não identificar melhoras nos níveis periodontais, diretamente, demonstrou uma ação positiva no controle da hemoglobina glicada, sendo uma possível terapia complementar para pacientes diabéticos.

\section{Discussão}

A ozonioterapia mostrou-se importante como tratamento adjuvante para diversas áreas da odontologia. Com foco na periodontia, estudos pré-clínicos demonstraram resultados satisfatórios desde as primeiras etapas do processo científico. Os efeitos in vitro foram apresentados nos estudos de Huth, et al (2007); Wang. et al (2018) e R. Niveda, Sankari Malaiappan (2019). Estes estudos apontaram efeitos significativos sobre as bacterias patogênicas, sobretudo nas colônias sensíveis à presença de oxigênio (microbiota principal para a progressão da doença periodontal). Ademais, todos os estudos demonstraram que o ozônio não apresentou nenhum efeito citotóxico às células humanas. O artigo de Wang et al (2018) comprovou uma ação positiva na produção de colágeno, em amostras submetidas a ozonioterapia, sugerindo, controle bacteriano, efeito benéfico para cicatrização tecidual. Entretanto, o trabalho de Skurska, et al (2010) não apresentou níveis significantes, entre a raspagem e alisamento coronoradicular com e, sem aplicação adjuvante da ozonioterapia; o estudo em questão, apresentou metodologia mista para avaliação de parâmetros clínicos e dos níveis in vitro das metaloproteinases. No entanto,os próprios autores ressaltaram a limitação da possível falha de aplicação direta do ozônio no local de efeito. Todos esses achados são elucidados pela revisão de Srikanth et al., (2013) que comprovou o potencial da terapia, como um adjuvante seguro e com alto potencial antimicrobiano. Os autores ressaltaram ainda, a falta de efeitos significativos para a utilização da ozonioterapia na clínica diária.

Em sequência, às etapas do processo científico, estudos em animais devem ser realizados para comprovar os efeitos 
terapêuticos. Entretanto, apenas o estudo de Saglam, et al (2019), apresentou essa metodologia. Para estes autores, o uso do ozônio contribuiu para a redução significativa de biomarcadores correlacionados com o processo de reabsorção óssea alveolar, atuando desta forma, para o controle da doença periodontal. Tais fatores podem ser explicados pela diminuição da carga microbiana local, assim como, pelo efeito estimulante do sistema circulatório e modulador do sistema imune. Achados demonstrados também na revisão de Srikanth et al., (2013).

Com relação aos efeitos clínicos, os estudos com tal desenho metodológico, resgatados na presente revisão, apresentaram divergências. Tais conflitos podem ser explicados pelas diferentes formas de aplicação da terapia com o ozônio ou mesmo, pelos efeitos esperados dos aspectos clínicos; os estudos em periodontia apresentam efeitos de mensuração extremamente precisos e, para alguns autores, os efeitos só representam significância, pela recuperação de milímetros de inserção óssea; outros, somente, investigam, a o grau da progressão da doença. A falta de padronização de desfechos, tanto impede a elaboração de uma revisão sistemática, quanto contribui, com uma falta de homogeneidade nos critérios de avaliação (Penteado et al 2010).

Diante do exposto, trabalhos clínicos que apresentaram desfechos positivos quanto ao uso da ozonioterapia, como adjuvante ao tratamento periodontal apresentaram características específicas. Ameyaroy et al., (2020) demonstraram, que houve melhora com relação aos níveis de inserção clínica com o uso da ozonioterapia, entretanto, é válido ressaltar que tal estudo utilizou como comparativo, a terapia fotodinâmica, ambos, em associação com o tratamento periodontal clássico, dessa forma, é imprudente associar esse resultado exclusivamente, com o uso da terapia complementar.

Hayakumo et al., (2013) comprovaram o efeito benéfico com relação ao uso do ozônio na forma de nanobolhas em comparação com o controle utilizando somente água. O estudo além de reforçar o efeito antimicrobiano da terapia, comprova que houve uma redução estatísticamente significativa do tamanho da bolsa e uma melhora nos níveis de inserção clínica. Apesar dos resultados promissores, é válido destacar que a amostra do estudo foi limitada (22 pacientes), podendo contribuir com resultados que não sejam generalizados para a população como um todo.

Talmaç, A. C., \& Çalişir, M. (2021) em estudo do tipo boca dividida comprovaram os efeitos da aplicação de ozônio gasoso com relação ao índice de tempo de sangramento gengival, apesar desse resultado animador, tal efeito pôde comprovar uma melhora nos parâmetros de cicatrização e ativação do sistema imune e não, necessariamente, uma correlação com os efeitos diretos na periodontite. Os demais parâmetros avaliados quando comparados entre controle e grupo de ozônio, não apresentaram efeito significativo.

Kaur et al., (2019) compararam os efeitos da água ozonizada com o gluconato de clorexidina a 0,2\% (principal agente utilizado para auxílio no controle da doença periodontal). Seus resultados demonstraram que ambas as terapias são eficazes na melhora de todos os parametros clínicos observados, entretanto, quando comparados os efeitos entre os dois tratamentos, a ozonioterapia apresentou superioridade com relação ao controle do biofilme periodontal. Dessa forma, sugere-se que a ozonioterapia tenha um potencial ainda maior em combinação com a raspagem e alisamento coronoradicular. $\mathrm{O}$ estudo, apesar de contar com uma amostra limitada (20 indivíduos), apresenta um resultado um pouco mais relevante em comparação com o de Hayakumo et al., (2013), uma vez que o trabalho de 2019, utiliza o modelo em boca dividida, aumentando o número de sítios observados e gerando um poder estatístico superior, dado o maior número de unidades amostrais. Ainda assim, ambos os estudos possuem limitação quanto a abrangência de seus resultados.

O estudo de Vasthavi et al., (2020), apesar de apresentar conclusões favoráveis ao uso da água ozonizada e uma metodologia robusta, apresenta falhas de interpretação de resultados expressiva. O estudo relata a melhora com a terapia, entretanto, nenhuma melhora estatisticamente representativa foi avaliada quando comparados, os grupos experimentais. Dessa forma, sua conclusão parece desenvolver uma narrativa com forte viés de tendência em pesquisa, possívelmente de publicação.

Nardi et al., (2020) buscaram os efeitos do uso de óleo de oliva ozonizado como forma de enxaguatório bucal em 
comparação com pacientes que não apresentaram terapia adjuvante. Tal estudo com metodologia de análise longitudinal acompanhou um n amostral significativo e avaliou significância estatística apenas com relação à diminuição dos nívels da metaloproteinase 8 (MMP-8). Tal redução é importante para a progressão da periodontite em virtude da associação com a atividade inflamatória, como enfatiza, Hiyari, S. et al., (2017).

Apesar de a literatura ser divergente quanto à eficácia da ozonioterapia, todos os artigos demonstraram ser segura a utilização desta terapia. Rapone et al., (2020) destaca que, apesar de os efeitos periodontais não serem expressivos de modo direto, a aplicação de ozônio de modo tópico, parece contribuir positivamente para o controle da hemoglobina glicada. Os níveis glicêmicos dos pacientes já são correlacionados com a saúde e recuperação periodontal, tendo, ambas as doenças, uma correlação estabelecida (Zainal et al, 2021).

Dengizek et al., (2019) afirma que o uso do ozônio não foi relevante quando comparado com a raspagem e alisamento coronário, isoladamente. Tal metodologia, no entanto, parece não compreender o uso da terapêutica como medida complementar ao tratamento mecânico. Tais considerações condizem com os achados de Skurska et al., (2010) e os de Tasdemir et al., (2019). Em todos os casos demonstrados, a ozonioterapia estava sendo comparada a curto prazo. Ademais, nenhum dos achados propõem que o uso da ozonioterapia pode substituir os procedimentos de raspagem mecânica profissional, dessa forma, a metodologia de comparação entre esses dois grupos, não parece identificar realmente o objetivo da terapêutica sugerida.

O relato de Domb WC (2014), pôde apontar para uma síntese dos estudos que surgiram a posteriori. Em 2014, o autor demonstrou que não eram homogêneos os relatos a respeito do uso da terapêutica complementar na periodontia, realidade que permanece constante. No entanto, a percepção das terapias integrativas e a sua compreensão de uso, podem estabelecer parâmetros que proporcionem estudos conjuntos, que permitam, de fato, aproximarem-se da realidade. Apesar das limitações do presente estudo, principalmente no que diz respeito à falta de homogeneidade de aplicação, concentração e metodologias de comparação entre os estudos, nota-se que a comparação metodológica coerente está relacionada à aplicação da ozonioterapia e de fármacos de terapêuticas adjuvantes, como a própria clorexidina.

\section{Conclusão}

A ozonioterapia poderá ser uma importante alternativa para o controle da periodontite quando associada à remoção mecânica do biofilme, como tratamento principal. Isto em virtude, de seus efeitos antibacterianos e cicatrizantes. Entretanto, a atual literatura não fornece bases sólidas para afirmar a sua real efetividade, frente a outras terapias auxiliares, já estabelecidas. Desta forma, para garantir uma escolha terapêutica baseada em evidências confiáveis, serão necessários mais estudos, com protocolos e metodologias padronizadas. A partir destes resultados, será possível fazer comparativos entre a utilização da ozonioterapia, em suas diferentes formas de aplicação, com outras terapêuticas adjuvantes, para o tratamento da periodontite.

\section{Referências}

Ameyaroy, D. K., Ramabhadran, B. K., Emmatty, R., Paul, T. P., \& Jose, P. (2020). Comparative evaluation of the effect of Ozone therapy and Photodynamic therapy in non-surgical management of Chronic periodontitis: A split mouth longitudinal study. Journal of Indian Society of Periodontology, $24(5), 447-453$. https://doi.org/10.4103/jisp.jisp_381_19

Anzolin, A. P., da Silveira-Kaross, N. L., \& Bertol, C. D. (2020). Ozonated oil in wound healing: what has already been proven? Medical gas research, 10(1), 54-59. https://doi.org/10.4103/2045-9912.279985

Deas, D. E., Moritz, A. J., Sagun, R. S., Jr, Gruwell, S. F., \& Powell, C. A. (2016). Scaling and root planing vs. conservative surgeryin the treatment of chronic periodontitis. Periodontology 2000, 71(1), 128-139. https://doi.org/10.1111/prd.12114

Deepthi, R., \& Bilichodmath, S. (2020). Ozone Therapy in Periodontics: A Meta-analysis. Contemporary clinical dentistry, 11(2), 108-115. https://doi.org/10.4103/ccd.ccd_79_19 
Domb W. C. (2014). Ozone therapy in dentistry. A brief review for physicians. Interventional neuroradiology: journal of peritherapeutic neuroradiology, surgical procedures and related neurosciences, 20(5), 632-636. https://doi.org/10.15274/INR-2014-10083

Fischer, R., Lira Junior, R., Retamal-Valdes, B., Figueiredo, L., Malheiros, Z., Stewart, B., \& Feres, M. (2020). Periodontal disease and its impact on general health in Latin America. Section V: Treatment of periodontitis. Brazilian Oral Research, 34(suppl 1). 10.1590/1807-3107bor-2020.vol34.0026

Graziani, F., Karapetsa, D., Alonso, B., \& Herrera, D. (2017). Nonsurgical and surgical treatment of periodontitis: how many options for one disease? Periodontology 2000, 75(1), 152-188. https://doi.org/10.1111/prd.12201

Gupta, G., \& Mansi, B. (2012). Ozone therapy in periodontics. Journal of medicine and life, 5(1), 59-67.

Hayakumo, S., Arakawa, S., Mano, Y., \& Izumi, Y. (2013). Clinical and microbiological effects of ozone nano-bubble water irrigation as an adjunct to mechanical subgingival debridement in periodontitis patients in a randomized controlled trial. Clinical oral investigations, 17(2), 379-388. https://doi.org/10.1007/s00784-012-0711-7

Herrera, D., Alonso, B., León, R., Roldán, S., \& Sanz, M. (2008). Antimicrobial therapy in periodontitis: the use of systemic antimicrobials against the subgingival biofilm. Journal of clinical periodontology, 35(8 Suppl), 45-66. https://doi.org/10.1111/j.1600-051X.2008.01260.x

Hiyari, S., Wong, R. L., Yaghsezian, A., Naghibi, A., Tetradis, S., Camargo, P. M., \& Pirih, F. Q. (2018). Ligature-induced peri- implantitis and periodontitis in mice. Journal of clinical periodontology, 45(1), 89-99. https://doi.org/10.1111/jcpe.12817

Honório, H., \& Santiago Júnior, J. (2021). Fundamentos das revisões sistemáticas em saúde (1st ed.). Santos Publicações.

Huth, K. C., Saugel, B., Jakob, F. M., Cappello, C., Quirling, M., Paschos, E., Ern, K., Hickel, R., \& Brand, K. (2007). Effect of aqueous ozone on the NFkappaB system. Journal of dental research, 86(5), 451-456. https://doi.org/10.1177/154405910708600512

Kaur, A., Bhavikatti, S. K., Das, S. S., Khanna, S., Jain, M., \& Kaur, A. (2019). Efficacy of Ozonised Water and 0.2\% Chlorhexidine Gluconate in the Management of Chronic Periodontitis when Used as an Irrigant in Conjugation with Phase I Therapy. The journal of contemporary dental practice, 20(3), 318323.

Kubyshkina K. P.., Podoprigora A. V., Oleynik O.I., Kharitonov Y. M. \& Belenova I. A. (2018). Statistical Evaluation Of Antimicrobial Influence Of Medical Ozone As A Part Of Inflammatory Prevention Of Periodontal Diseases. RJPBCS, 9(6), 690-696

Nardi, G. M., Cesarano, F., Papa, G., Chiavistelli, L., Ardan, R., Jedlinski, M., Mazur, M., Grassi, R., \& Grassi, F. R. (2020). Evaluation of Salivary Matrix Metalloproteinase (MMP-8) in Periodontal Patients Undergoing Non-Surgical Periodontal Therapy and Mouthwash Based on Ozonated Olive Oil: A Randomized Clinical Trial. International journal of environmental research and publichealth, 17(18), 6619. https://doi.org/10.3390/ijerph17186619

Niveda R. \& Malaiappan S. (2019). Comparison of the amount of bacterial microcolonies formed on using distilled water and ozonated water as an irrigating agent. Drug invention today, 11(4), 1605-1609.

Oliveira, J. K. S. de, Carvalho, J. M. de, Silva, C. A., Lima, M. B. de, Cavalcante, P. M. F., \& Paiva, D. F. F. (2020). Relação entre aexposição ao pesticida Rotenona e o desenvolvimento de sintomas motores e não-motores da doença de Parkinson. Research, Society and Development, 9(9), e706997917. https://doi.org/10.33448/rsd-v9i9.7917

Papapanou, P. N., et al. (2018). Periodontitis: Consensus report of workgroup 2 of the 2017 World Workshop on the Classification of Periodontal and PeriImplant Diseases and Conditions. Journal of clinical periodontology, 45 Suppl 20, S162-S170. https://doi.org/10.1111/jcpe.12946

Penteado, L. A. M., Awabdi, T. W. M., Auto, V. C., Rodrigues, E. D. O., Oliveira, D. P.\& Santos, N. B. (2010). Avaliação da padronização das sondas periodontais utilizadas em uma instituição de ensino superior de Maceió-AL. Int J Dent, 9(3), 120-127.

Rapone, B., Ferrara, E., Corsalini, M., Converti, I., Grassi, F. R., Santacroce, L., Topi, S., Gnoni, A., Scacco, S., Scarano, A., \& Delvecchio, M. (2020). The Effect of Gaseous Ozone Therapy in Conjunction with Periodontal Treatment on Glycated Hemoglobin.

Level in Subjects with Type 2 Diabetes Mellitus: An Unmasked Randomized Controlled Trial. International journal of environmental research and public health, 17(15), 5467. https://doi.org/10.3390/ijerph17155467

Saglam, E., Alinca, S., Celik, T., Hacisalihoglu, U., \& Dogan, M. (2020). Evaluation of the effect of topical and systemic ozone application in periodontitis: an experimental study in rats. Journal Of Applied Oral Science, 28. 10.1590/1678-7757-2019-0140

Seydanur Dengizek, E., Serkan, D., Abubekir, E., Aysun Bay, K., Onder, O., \& Arife, C. (2019). Evaluating clinical and laboratory effects of ozone in nonsurgical periodontal treatment: a randomized controlled trial. Journal Of Applied Oral Science, 27. 10.1590/1678-7757-2018-0108

Skurska, A., Pietruska, M. D., Paniczko-Drężek, A., Dolińska, E., Zelazowska-Rutkowska, B., Zak, J., Pietruski, J., Milewski, R., \& Wysocka, J. (2010). Evaluation of the influence of ozonotherapy on the clinical parameters and MMP levels in patients with chronic and aggressive periodontitis. Advances in medical sciences, 55(2), 297-307. https://doi.org/10.2478/v10039-010-0048-X

Slots J. (2017). Periodontitis: facts, fallacies and the future. Periodontology 2000, 75(1), 7-23. https://doi.org/10.1111/prd.12221

Srikanth, A., Sathish, M., \& Sri Harsha, A. V. (2013). Application of ozone in the treatment of periodontal disease. Journal of pharmacy \& bioallied sciences, 5(Suppl 1), S89-S94. https://doi.org/10.4103/0975-7406.113304

Suh, Y., Patel, S., Kaitlyn, R., Gandhi, J., Joshi, G., Smith, N. L., \& Khan, S. A. (2019). Clinical utility of ozone therapy in dental and oral medicine. Medical gas research, 9(3), 163-167. https://doi.org/10.4103/2045-9912.266997

Talmaç, A. C., \& Çalişir, M. (2021). Efficacy of gaseous ozone in smoking and non-smoking gingivitis patients. Irish journal of medical science, 190(1), 325333. https://doi.org/10.1007/s11845-020-02271-x 
Research, Society and Development, v. 10, n. 15, e278101522961, 2021

(CC BY 4.0) | ISSN 2525-3409 | DOI: http://dx.doi.org/10.33448/rsd-v10i15.22961

Tasdemir, Z., Oskaybas, M. N., Alkan, A. B., \& Cakmak, O. (2019). The effects of ozone therapy on periodontal therapy: A randomized placebo-controlled clinical trial. Oral diseases, 25(4), 1195-1202. https://doi.org/10.1111/odi.13060

Uraz, A., Karaduman, B., Isler, S., Gönen, S., \& Çetiner, D. (2019). Ozone application as adjunctive therapy in chronic periodontitis: Clinical, microbiological and biochemical aspects. Journal Of Dental Sciences, 14(1), 27-37. 10.1016/j.jds.2018.06.005

Vasthavi, C., Babu, H. M., Rangaraju, V. M., Dasappa, S., Jagadish, L., \& Shivamurthy, R. (2020). Evaluation of ozone as an adjunct to scaling and root planing in the treatment of chronic periodontitis: A randomized clinico-microbial study. Journal of Indian Society of Periodontology, 24(1), 42-46. https://doi.org/10.4103/jisp.jisp_162_19

Wang, P., Tachi, Y., Masuno, K., Okusa, N., \& Imamura, Y. (2018). The Study of Ozone Ointment on Human Gingival Fibroblasts Cell Proliferation Ability and Anti-Inflammatory. Journal Of Hard Tissue Biology, 27(3), 209-212. 10.2485/jhtb.27.209

Y1lmaz, S., Algan, S., Gursoy, H., Noyan, U., Kuru, B. E., \& Kadir, T. (2013). Evaluation of the clinical and antimicrobial effects of the Er:YAG laser or topical gaseous ozone as adjuncts to initial periodontal therapy. Photomedicine and laser surgery, 31(6), 293- 298. https://doi.org/10.1089/pho.2012.3379 